\title{
Modified gracilis muscle transposition for fecal incontinence
}

\author{
H. J. Yang ${ }^{1} \cdot$ Y. W. Li $^{1} \cdot$ Z. X. Zhang ${ }^{1}$
}

Received: 12 February 2020 / Accepted: 21 February 2020 / Published online: 4 March 2020

(c) Springer Nature Switzerland AG 2020

Fecal incontinence is one of the most psychosocially disabling conditions in a healthy person. It can lead to depression, social isolation, loss of self-confidence and self-esteem. Skeletal muscle transplantation has proved to be effective in treating end-stage anal incontinence [1,2]. Gracilis muscle transplantation, first described by Pickrell et al. in 1952, is the preferred muscle for skeletal muscle transplantation because of the minimal impact on donor function [3]. In traditional gracilis muscle transposition, the distal tendon of the muscle was fixed to the contralateral ischial tuberosity periosteum as a neosphincter to augment or replace the anal sphincter. Several studies have found that this was efficient, nevertheless, evacuatory dysfunction is a common problem due to excessive stretching of the transposed gracilis tendon [4]. In our department, the procedure is modified and simplified, with the distal tendon fixed to the ipsilateral ischial tuberosity periosteum to reduce the risk of postoperative anal stenosis. The newly transplanted muscle strengthens part of the puborectalis muscle to the attached video demonstrates the surgical procedure of modified gracilis muscle transposition step by step.

The operation was carried out in lithotomy position. Gracilis muscle flap was isolated through two incisions on the medial side of the right thigh, and was wrapped around the anal canal, attached to the ipsilateral ischial tuberosity periosteum through two incisions on the perineum. The muscle was always long enough to wrap around the anus and be attached to the ipsilateral ischial tuberosity and, therefore, would not lead to neosphincter stenosis. The procedure is simplified and involves at least one incision less. Complete continence to liquids and solids was obtained.

\section{Compliance with ethical standards}

Conflict of interest The authors declare that they have no conflict of interest.

Ethical approval All human studies have been reviewed by the appropriate ethics committee and have therefore been performed in accordance with the ethical standards laid down in an appropriate version of the 1965 Declaration of Helsinki.

Informed consent Informed consent was obtained from all individual participants included in the study.

\section{References}

1. Hassan MZ, Rathnayaka MM, Deen KI (2010) Modified dynamic gracilis neosphincter for fecal incontinence: an analysis of functional outcome at a single institution. World J Surg 34(7):1641-1647

2. Chandra A, Mishra B, Kumar S et al (2019) Composite antropyloric valve and gluteus maximus muscle wrap for neoanal reconstruction: initial results. Dis Colon Rectum 62(1):104-111

3. Pickrell KL, Broadbent TR, Masters FW, Metzger JT (1952) Construction of a rectal sphincter and restoration of anal continence by transplanting the gracilis muscle; a report of four cases in children. Ann Surg 135(6):853-862

4. Cera SM, Wexner SD (2005) Muscle transposition: does it still have a role? Clin Colon Rectal Surg 18(1):46-54

Publisher's Note Springer Nature remains neutral with regard to jurisdictional claims in published maps and institutional affiliations.

Z. X. Zhang

zhangzuoxing@163.net

1 Department of Colorectal Surgery, Tianjin Union Medical

Center, Tianjin 300000, China 